\title{
Prognostic value of postoperative proteinuria for predicting early renal outcome after kidney transplantation
}

\author{
Kyungho Park ${ }^{1}$, Mee Yeon Park ${ }^{1}$, Hyun Suk Lee ${ }^{1}$, Junseok Jeon ${ }^{1}$, Kyo Won Lee ${ }^{2}$, Jung Eun Lee ${ }^{1}$, Jae Berm Park², Wooseong \\ Huh $^{1}$, Yoon Goo Kim ${ }^{1}$, Hye Ryoun Jang ${ }^{1}$ \\ ${ }^{1}$ Division of Nephrology, Department of Internal Medicine, Samsung Medical Center, Seoul, Korea \\ ${ }^{2}$ Division of Transplantation, Department of Surgery, Samsung Medical Center, Seoul, Korea
}

Background: Proteinuria in kidney transplant recipients (KTRs) was reported to predict poor clinical outcomes. In this single center retrospective cohort study, we investigated the prognostic value of spot urine protein to creatinine ratio (PCR) or albumin to creatinine ratio (ACR) during early postoperative period.

Methods: A total of 353 KTRs with urine PCR or ACR data within postoperative day 7 after KT between 2014 and 2017 were included in the final analyses. PCR and ACR were serially followed up during the immediate postoperative period (within postoperative day 7), before discharge (postoperative 2-3 weeks), and at postoperative 3-6 months. Primary and secondary outcomes were estimated glomerular filtration rate (eGFR) at 1 year after KT and the incidence of delayed graft function (DGF), respectively. Results: After adjusting for sex, age, donor status, and acute rejection within 3 months, KTRs with higher PCR ( $\beta$ coefficient=-8.66, $\mathrm{P}=0.01$ ) or ACR ( $\beta$ coefficient=-6.97, $\mathrm{P}<0.001)$ at postoperative $3-6$ months showed lower eGFR at 1 year after $\mathrm{KT}$. Deceased donor kidney transplantation (DDKT) recipients with immediate postoperative PCR $\geq 3 \mathrm{mg} / \mathrm{mg}$ showed higher incidence of DGF (PCR $<3 \mathrm{mg} / \mathrm{mg}$ vs. PCR $\geq 3 \mathrm{mg} / \mathrm{mg}, 12 \%$ vs. 39\%; $\mathrm{P}<0.001)$ and lower eGFR before discharge (64.2 [range, $49.7-$ 85.4 ] vs. 49.6 [range, 35.8-66.5]; $P=0.001$ ) compared to KTRs with immediate postoperative $P C R<3$ mg/mg.

Conclusions: Proteinuria at postoperative 3-6 months was identified as a potential surrogate marker predicting early renal outcome after KT. Heavy proteinuria during the immediate postoperative period may be a potential predictor of DGF in DDKT recipients. Our study suggests that early postoperative proteinuria may be a useful biomarker for predicting early renal outcome after KT.

Corresponding author: Kyungho Park

E-mail: ds3ixc@gmail.com

(C) The Korean Society for Transplantation

This is an Open Access article distributed under the terms of the Creative Commons Attribution Non-Commercial License (http://creativecommons.org/licenses/by-nc/4.0/) which permits unrestricted non-commercial use, distribution, and reproduction in any medium, provided the original work is properly cited. 